\title{
Experimental Parity-Time Symmetric Quantum Walks for Centrality Ranking on Directed Graphs
}

\author{
Tong Wu (吴通)๑, ${ }^{1}$ J. A. Izaac $\odot,{ }^{2}$ Zi-Xi Li (黎子溪)๑, ${ }^{1}$ Kai Wang (王凯) ${ }^{1}$ Zhao-Zhong Chen (陈召忠), ${ }^{1}$ \\ Shining Zhu (祝世宁), ${ }^{1}$ J. B. Wang, ${ }^{2}$ and Xiao-Song Ma (马小松) ${ }^{1, *}$ \\ ${ }^{1}$ National Laboratory of Solid State Microstructures, School of Physics, Collaborative Innovation Center of Advanced Microstructures, \\ Nanjing University, Nanjing 210093, China \\ ${ }^{2}$ School of Physics, The University of Western Australia, Perth, Western Australia 6009, Australia
}

(Received 17 December 2019; accepted 28 October 2020; published 8 December 2020)

\begin{abstract}
Using quantum walks (QWs) to rank the centrality of nodes in networks, represented by graphs, is advantageous compared to certain widely used classical algorithms. However, it is challenging to implement a directed graph via QW, since it corresponds to a non-Hermitian Hamiltonian and thus cannot be accomplished by conventional QW. Here we report the realizations of centrality rankings of a three-, a four-, and a nine-vertex directed graph with parity-time $(P T)$ symmetric quantum walks by using highdimensional photonic quantum states, multiple concatenated interferometers, and dimension dependent loss to achieve these. We demonstrate the advantage of the QW approach experimentally by breaking the vertex rank degeneracy in a four-vertex graph. Furthermore, we extend our experiment from single-photon to two-photon Fock states as inputs and realize the centrality ranking of a nine-vertex graph. Our work shows that a $P T$ symmetric multiphoton quantum walk paves the way for realizing advanced algorithms.
\end{abstract}

DOI: $10.1103 /$ PhysRevLett.125.240501

Introduction.-Classical random walks are of fundamental importance in a variety of scientific disciplines, including physics, biology, and economics. Quantum walks (QWs), first introduced by Aharonov et al. [1], are the quantum analog of classical random walks [2]. Quantum walks harness the superposition and the entanglement of quantum systems, which lead to many applications in quantum information, including the development of quantum-enhanced algorithms [3-6], quantum simulations [7-11], and universal quantum computation [12,13]. Quantum walks have been realized in many physical systems, including photons [14-26] and atoms [27-31]. See the review in Ref. [32].

One promising application of quantum walks is to provide an efficient quantum algorithm for vertex centrality ranking in network analysis [6,33-37]. Using continuous-time quantum walks (CTQWs), the aforementioned algorithm has been implemented on an undirected graph [10]. However, in most applications, one needs to analyze directed graphs, which are more general than undirected graphs in representing practical systems. For instance, in the classical PAGERANK algorithms, revealing the relevance between websites and listing their ranks requires network analysis on directed graphs. It is challenging to rank the centrality of a directed graph with $\mathrm{QW}$ experimentally, which stems from the intrinsic conflict between the directionality of the PAGERANK algorithm and the nondirectionality or unitarity of traditional QWs. This issue has been theoretically solved by introducing pseudo-Hermitian evolutions $[11,38,39]$. In this work, we demonstrate the first realization of quantum walks on directed graphs with photons and their applications in centrality ranking.

Quantum walk on a three-vertex directed graph.-As shown in Fig. 1(a), a network can be represented as a graph $G(V, E)$ consisting of vertices $j \in V$ and edges $(i, j) \in E$. The adjacency matrix $A$ is used to characterize the graph $G$, defined as

$$
A_{i j}= \begin{cases}1 & (i, j) \in E \\ 0 & (i, j) \notin E .\end{cases}
$$

In a directed (undirected) graph, the adjacency matrix $A$ is unsymmetric (symmetric) where $A_{i j} \neq A_{j i}\left(A_{i j}=A_{j i}\right)$. By using classical algorithms, such as PAGERANK, one can calculate the centrality ranking of the graph representing the network, as shown in Fig. 1(b). One can also use quantum algorithms, such as quantum walk, to rank the centrality of each vertex, as shown in Fig. 1(c). For a CTQW on graph $G$, the conjugate transpose of the Hamiltonian is the graph Laplacian of the adjacency matrix and can be written as $[1,2,32]$

$$
\left(H^{\dagger}\right)_{i j}=\mathcal{L}_{i j}=\left(\sum_{k} A_{i k}\right) \delta_{i j}-A_{i j}
$$

By solving the Schrödinger equation, one obtains the time evolution of the walker and its wave function, at time $t$, with $|\psi(t)\rangle=e^{-i H t}|\psi(0)\rangle$. In our work, we prepare an 


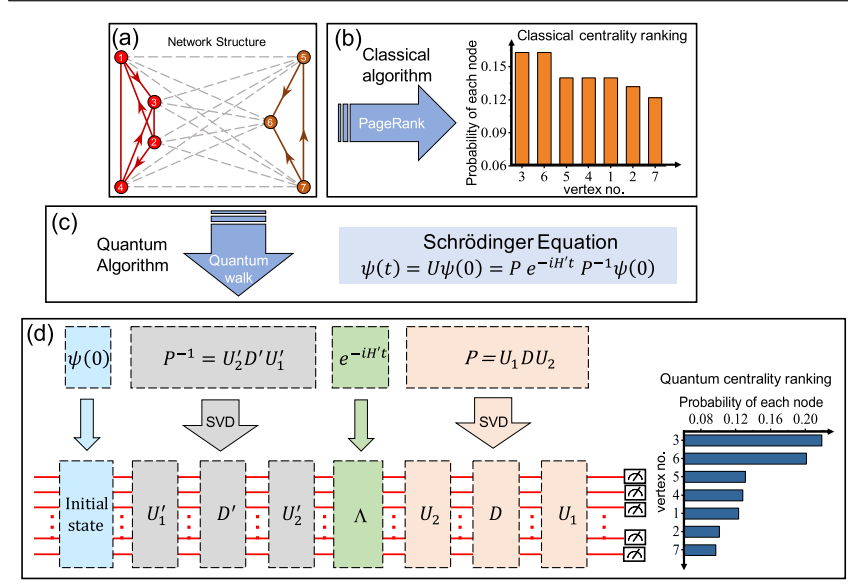

FIG. 1. Network analysis with classical and quantum algorithms. (a) A general network structure is represented as a directed graph with weighted edges, which are characterized by its adjacency matrix. (b) By using the classical PAGERANK algorithm, one can calculate the centrality ranking of the graph representing the network. (c) In the quantum scenario, one can map the centrality ranking problem to solve quantum walk dynamics with Schrödinger equation. The dynamics are governed by the time-evolution operator $U$, which is related to the adjacency matrix of the graph (see text). By diagonalizing the Hamiltonian, one decomposes $U$ into three parts, the diagonal $H^{\prime}$ and two matrices $P$ and $P^{-1}$ for a general graph. (d) The recipe for performing the centrality ranking of a graph with quantum walks. We first generate the initial state $|\psi(0)\rangle$, whose dimensionality corresponds to the number of vertices in the graph, represented by the red paths. Then we use singular value decomposition (SVD) to decompose $P^{-1}$ and $P$ into $P^{-1}=$ $U_{2}^{\prime} D^{\prime} U_{1}^{\prime}$ and $P=U_{1} D U_{2}$. The evolution of diagonalized Hamiltonian $H^{\prime}$ can be realized with matrix $\Lambda$. Finally we measure the output from this circuitry and obtain the centrality of the vertex of the graph.

initial state and then let it evolve as a CTQW, where the time variable $t$ is continuous. Experimentally, we use the phase introduced by wave plates, which can be tuned continuously to mimic the continuous-time evolution of single-photon states. On a directed graph, its adjacency matrix $A$ is no longer symmetric and hence the Hamiltonian $H$ is not Hermitian. Consequently, the time-evolution operator is not unitary and the squared norm of $|\psi(t)\rangle$ could grow or decay exponentially. Note that there have been proposals focusing on the nonunitary evolution, for example, Szegedy quantum walks $[25,34,40]$ and open quantum walks $[11,35,36]$. Here we follow the theoretical model proposed in Ref. [11] and experimentally realize a quantum walk on directed graphs. In Fig. 1(d), we show the recipe for performing the centrality ranking of a general graph with quantum walks. Firstly, we generate a highdimensional initial state, $|\psi(0)\rangle$. Its dimensionality corresponds to the number of vertices in the graph of interest. The Hamiltonian $H$ can be diagonalized and decomposed into three matrices: $P^{-1}, H^{\prime}$, and $P$. If the graph is directed, both $P^{-1}$ and $P$ are not necessarily unitary. Moreover, if the eigenvalues of $H$ are all real and the eigenvectors are linearly independent, $H$ is pseudo-Hermitian [41]. This is the focus throughout this work. We then use the singular value decomposition (SVD) to decompose $P^{-1}$ and $P$ into $P^{-1}=U_{2}^{\prime} D^{\prime} U_{1}^{\prime}$ and $P=U_{1} D U_{2}$, where $D^{\prime}(D)$ is a diagonal matrix with non-negative real numbers on the diagonal, which are known as the singular values of $P^{-1}$ $(P)$. The evolution of diagonalized Hamiltonian $H^{\prime}$ can be realized with matrix $\Lambda$, representing the unitary dynamics. Finally, we measure the output from this circuitry and obtain the centrality of the vertex of the graph.

In this work, we use three simple yet illustrative exemplary graphs to demonstrate the abovementioned concept. The first one is a three-vertex directed graph shown in Fig. 2(a), whose adjacency matrix and the corresponding Hamiltonian are

$$
A_{3}=\left[\begin{array}{lll}
0 & 1 & 0 \\
1 & 0 & 0 \\
1 & 1 & 0
\end{array}\right], \quad H_{3}=\left[\begin{array}{ccc}
1 & -1 & -1 \\
-1 & 1 & -1 \\
0 & 0 & 2
\end{array}\right] .
$$

We can easily verify that $H_{3}$ is pseudo-Hermitian [41]. Although the time evolution is not unitary and hence the total probability is not conserved, the pseudo-Hermitian character of the system ensures that the total probability of the quantum walker's state will just oscillate, with no exponential growth and decay.

We decompose the time-evolution operator such that the time dependence is restricted to a single diagonal unitary matrix. We can diagonalize $U$ and obtain the time-evolution operator as

$$
\begin{aligned}
U & =P \Lambda P^{-1} \\
& =\frac{1}{2}\left[\begin{array}{ccc}
-1 & 1 & -1 \\
1 & 1 & 0 \\
0 & 0 & 1
\end{array}\right]\left[\begin{array}{ccc}
e^{-2 i t} & 0 & 0 \\
0 & 1 & 0 \\
0 & 0 & e^{-2 i t}
\end{array}\right]\left[\begin{array}{ccc}
-1 & 1 & -1 \\
1 & 1 & 1 \\
0 & 0 & 2
\end{array}\right] .
\end{aligned}
$$

The diagonal matrix $\Lambda$ is unitary and represents the phase accumulation in CTQW, which can be implemented experimentally, as shown in Sec. III of Ref. [42]. However, the two outer matrices, $P$ and $P^{-1}$, are not unitary, and are nontrivial to realize experimentally with linear optics. We overcome this challenge by expanding the dimensionality of states that the single photon occupies and introducing nonunitary loss. Firstly, we employ a ququart, whose Hilbert space is four dimensional and is spanned by its horizontal and vertical polarization and two optical paths $(|1 H\rangle,|1 V\rangle,|2 H\rangle,|2 V\rangle)$. Secondly, we use dimensiondependent photon-number loss. Finally, we verify the nonunitary evolution for three out of four dimensions by obtaining the probability for each vertex. See Sec. IV of Ref. [42] for details on the realizations. 
(a)

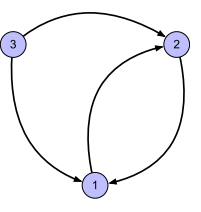

(b)

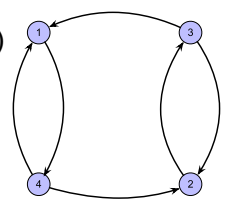

(c)

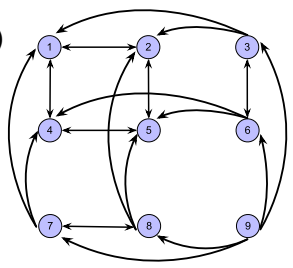

(d)

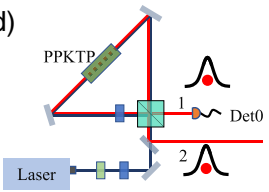

(e)

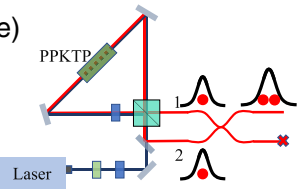

(f)
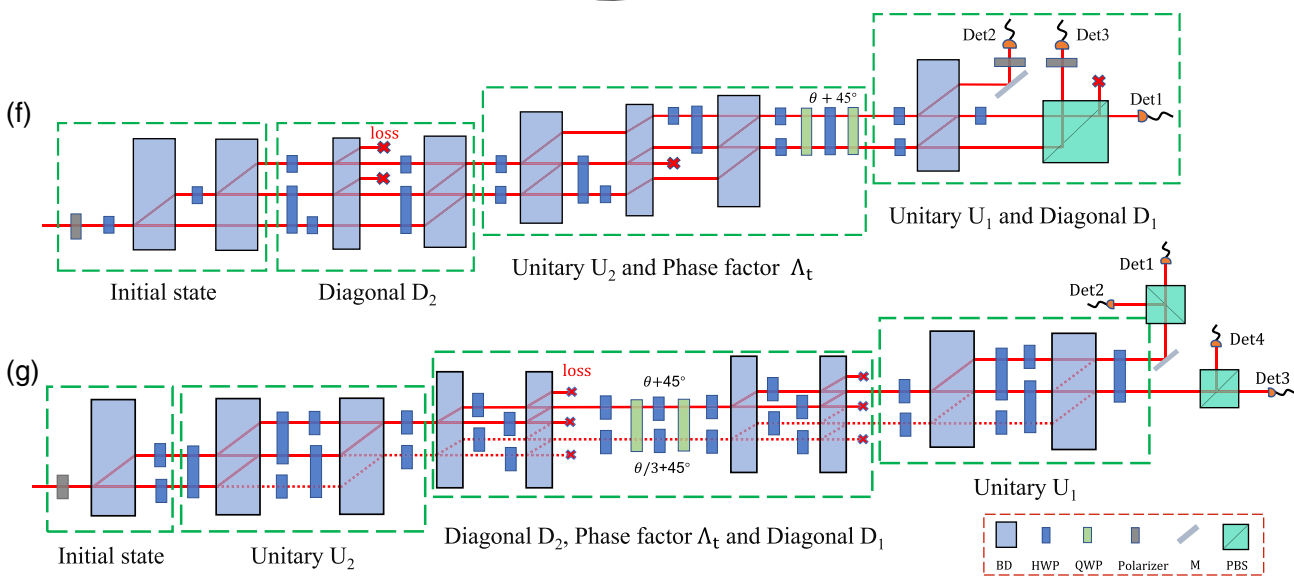

FIG. 2. Realizing quantum walk for centrality ranking on directed graphs with photonic Fock states. The vertex centrality rankings of a three-vertex (a), a four-vertex (b), and a nine-vertex (c) directed graph are realized in our experiment. (d) The single-photon source. A pair of photons (photons 1 and 2) are generated from the spontaneous parametric down-conversion process, which is realized by embedding a PPKTP crystal in a Sagnac interferometer. We couple the photons into single-mode fibers, respectively. Photon 1 is detected by Det0, which is a single-photon avalanche diode (SPAD). The single-photon count rate of photon 1 is about $600 \mathrm{kHz}$. This detection heralds the presence of photon 2. (e) The two-photon source. We interfere photons 1 and 2 on a fiber beam splitter (BS). Because of bosonic nature of single photons, they will bunch and exhibit Hong-Ou-Mandel interference. Therefore, they exit together in the same spatial mode of the BS with a count rate of about $8.5 \mathrm{kHz}$. Then we send the two-photon state into the circuitry. Panels (f) and (g) show the optical circuitries for realizing the three-vertex and four-vertex directed quantum walk. To realize the continuous quantum walk on a directed graph, photon 2 is sent through an optical network consisting of beam displacers (BDs), polarizing beam splitters (PBSs), half-wave plates (HWPs), quarter-wave plates (QWPs), polarizers and mirrors (M). This optical network can be divided into several blocks, corresponding to diagonal matrix $D_{2}$, unitary matrix $U_{2}$, evolutionary matrix $\Lambda_{t}$, unitary matrix $U_{1}$, and diagonal matrix $D_{1}$. The optical transmissions of the three-vertex circuitry and the four-vertex circuitry are about $1.5 \%-4.6 \%$ and $1.9 \%-2.5 \%$, respectively. For the nine-vertex graph, we use the circuitry in (f) in conjunction with nondeterministic photon-number resolving detections, which are realized by using three fiber BSs and six SPADs (not shown).

In Fig. 2, we show our experimental schematic of realizing quantum walks on directed graphs. We create photon pairs using type-II spontaneous parametric downconversion in a 20 -mm-long periodically poled potassium titanyl phosphate (PPKTP) crystal, embedded in a Sagnac interferometer [49,50]. The $405 \mathrm{~nm}$ laser is set to vertical polarization so that only one arm of the Sagnac interferometer is pumped. We use heralded single-photon Fock states as inputs for the three- [Fig. 2(a)] and four-vertex [Fig. 2(b)] graphs. As shown in Fig. 2(d), in order to create a single-photon Fock state, we detect photon 1 by Det0, which serves as a trigger and heralds the existence of photon 2. We realize the CTQW on the three-vertex directed graph as shown in Fig. 2(a) with a single-photon ququart. It is worth noting that one can use classical laser to emulate the results we obtained with the heralded single photons. However, genuine multipartite entangled states are required for realizing large-scale complex network analysis, which cannot be realized with attenuated laser beam.
Based on the decomposition methods discussed Sec. IV of Ref. [42], we employ an optical circuitry consisting of beam displacers (BDs), half-wave plates (HWPs), and quarter-wave plates (QWPs) to manipulate the path and polarization states of photon 2 , as shown in Fig. 2(f). Because of the stability of the concatenated interferometer made by BDs, after an initial phase calibration with classical light, we are able to obtain good results over a few hours without active feedback control. The details of realizing diagonal matrices $\left(D_{1}\right.$ and $\left.D_{2}\right)$ and unitary matrices $\left(U_{1}\right.$ and $\left.U_{2}\right)$ can be found in Secs. I and II of Ref. [42]. Note that we have stopped the photon occupying mode $|2 V\rangle$ after the dimensionality expansion in the step of evolution matrix $\Lambda_{t}$ and $D_{1}$, respectively. The coincidence detection events of photons at detector Det0 and Det1, Det0 and Det2, Det0 and Det3 (all with a 2 ns coincidence time window) indicate successful runs of the directed quantum walks and correspond to the centrality probabilities of vertices 1,2 , and 3 , respectively. We show the raw data of these three coincidence counts as a function of the angle of 


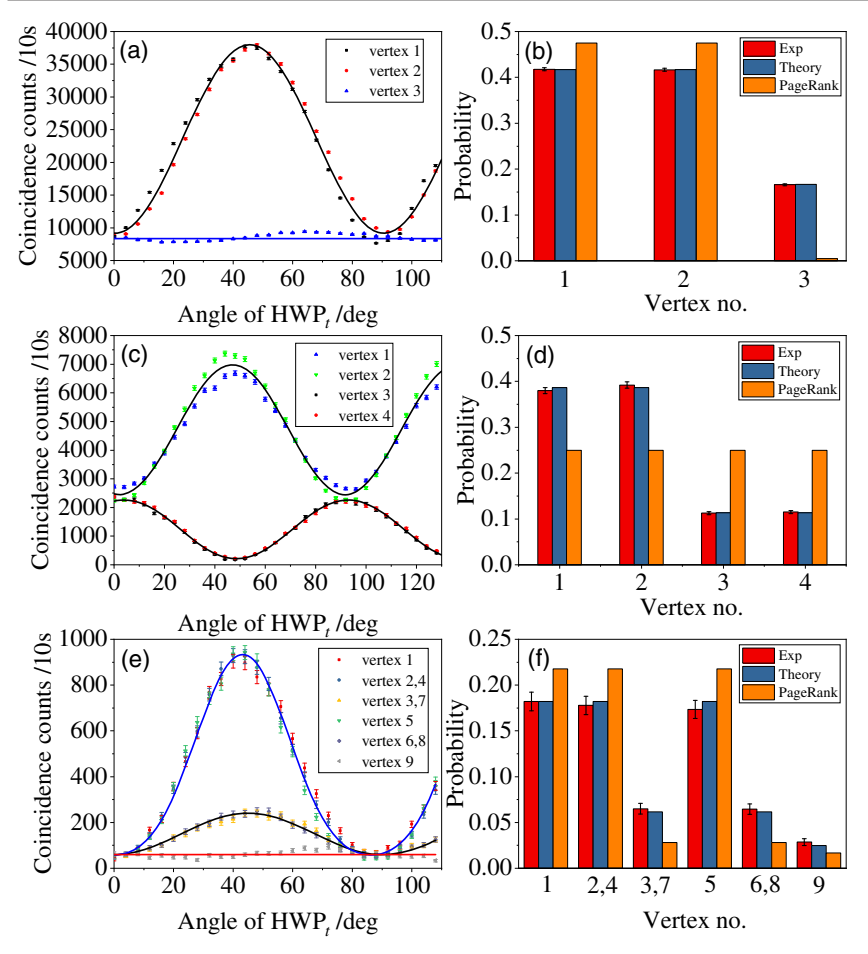

FIG. 3. Experimental data. (a) The black, red, and blue data points are the experimental coincidence counts between Det0 and Det1, Det0 and Det2, and Det0 and Det3 as a function of the rotation angle of $\mathrm{HWP}_{t}$, respectively. The black curve is the average of the fits to black and red data points. (b) The red and blue bars represent the probabilities of photon 2 occupying each vertex averaging over a $2 \pi$ period, which correspond to the centrality ranking of vertex 1,2 , and 3 shown in Fig. 2(a). The orange bars indicate the centrality calculated using the classical PAGERANK algorithm. (c) The blue, green, black, and red data points are the experimental coincidence counts between Det0 and Det1, Det0 and Det2, Det0 and Det3, and Det0 and Det4 as a function of the rotation angle of $\mathrm{HWP}_{t}$, respectively. The two black curves are the average of the fits to blue and green and the black and red data points, respectively. (d) The centrality ranking of vertex 1-4 in Fig. 2(b). (e) The red, blue, yellow, green, purple, and gray data points are the experimental coincidence counts for the nine-vertex graph. The blue, black, and red curves are the fits to the corresponding data points. (f) The centrality ranking of vertex 1-9 in Fig. 2(c). The uncertainties represent standard deviations deduced from propagated Poissonian statistics.

$\mathrm{HWP}_{t}$ in Fig. 3(a). By rotating this angle, we can adjust the phase between the horizontal and vertical polarization states of photon 2. This birefringent phase corresponds to the evolution time of the system, as shown in Eq. (5). It is clear to see that the coincidence counts of Det0 and Det1, and Det0 and Det2, depend strongly on the angle of $\mathrm{HWP}_{t}$. By contrast, the coincidence counts of Det 0 and Det 3 are much less sensitive to the angle of $\mathrm{HWP}_{t}$. By taking the average values of these three coincidence counts and normalizing them to the sum of these three average values, we obtain the experimental centralities of each vertex respectively, shown in Fig. 3(b).
Quantum walk on a four-vertex directed graph.-A CTQW based algorithm is more advantageous for certain graphs compared to the classical PAGERANK algorithm in terms of lifting the degeneracy of centralities of vertices. Surprisingly, this quantum advantage can already be found in a small-scale graph with four vertices, as shown in Fig. 2(b). In this case, vertices 1 and 2, and vertices 3 and 4 , are two sets of equivalent vertices. However, if we use the classical PAGERANK algorithm to analyze this graph, we will find that all four vertices have the same centralities, which are incorrect. If we use the CTQW, we can break this four-vertex degeneracy and achieve the correct centralities ranking of vertices. Particularly, we will show that, by using CTQW, the centralities of vertices 1 and 2 are higher than that of vertices 3 and 4, which is the truth of this graph [Fig. 2(b)].

The details of the four-vertex, such as adjacency matrix, and the decomposition of its time-evolution operator can be found in Sec. V of Ref. [42]. In Fig. 2(g), we show the experimental setup for realizing quantum walks on the four-vertex directed graph. The initial state of photon 2 is the equally weighted superposition state of the four modes $(|1 H\rangle,|1 V\rangle,|2 H\rangle,|2 V\rangle)$. The photons travel in the solid lines, and there is no photon passing through the dotted lines. We show the experimental data of the four coincidence counts in Figs. 3(c) and 3(d). The results of the CTQW centrality test agree well with theory, ranking vertices 1 and 2 above vertices 3 and 4 . We found the degeneracy breaking by using a $P T$ symmetric quantum walk is quite general in the sense that many more examples are readily found, which have been explained in Sec. VI of Ref. [42].

Quantum walk on a nine-vertex directed graph with twophoton Fock state.-To extend the vertices and hence the size of the graph of interests, one needs to increase the dimensionality of the experimental system. The number of the vertices in the graph grows exponentially with the number of the photons. If the local dimension of each photon is $d$ and the photon number is $n$, then the total number of the vertices in the graph is $d^{n}$. By using two identical single photons as input, we increase the dimensionality of our system from three to nine, which corresponds to the directed graph in Fig. 2(c). See Sec. VII of Ref. [42] for details of its Hamiltonian. In order to obtain the required two-photon Fock state, we employ the HongOu-Mandel interference [51] with the photon pairs generated from the spontaneous parametric down-conversion (SPDC) source in a fiber beam splitter (Sec. VIII of Ref. [42]). By doing so, we create two identical photons with the same polarization on the same path, as shown in Fig. 2(e). In order to resolve the photon number occupying the same mode, we use fiber beam splitters and coincidence detection to implement nondeterministic photon-number resolving detection (not shown) [52]. By concatenating these setups, we are able to simulate the evolution dynamics of a two-photon Fock state walking on a directed graph [shown 
in Fig. 3(e)] and obtain the results of centrality ranking among these nine vertices [shown in Fig. 3(f)], which agree well with the theory. In our experimental realization, due to the probabilistic nature of photon-number resolving detection, the coincidence counts of the same polarization in the same mode is about half of that with different polarization in the same or different modes. For instance, the coincidence counts of $|1 H 1 H\rangle$ is about half of that of $|1 H 1 V\rangle$. Therefore, we take the averages of the coincidence counts $|1 H 1 V\rangle$ and $|1 V 1 H\rangle,|2 H 2 V\rangle$ and $|2 V 2 H\rangle$, and $|3 H 3 V\rangle$ and $|3 V 3 H\rangle$, respectively.

Conclusion.-In this study, we have experimentally realized a quantum walk on directed graphs with genuine photonic Fock states. The required nonunitary evolution of the walker on the directed graph is decomposed into a series of unitary and nonunitary transformations in high-dimensional Hilbert spaces spanned by the polarization and spatial modes of photons. We successfully demonstrated quantum centrality rankings on a three-vertex and a ninevertex graph with $P T$ symmetric quantum walks, and the advantage of the QW approach by breaking the vertex rank degeneracy in the four-vertex graph compared with the classical PageRAnK algorithm. Our work shows that, in conjunction with unitary evolutions, the controllable loss can be employed as a crucial degree of freedom in quantum walks and may be extended to other quantum information tasks. In order to implement scalable quantum walks for large networks, one needs to generate high-quality single photons [53] as well as to realize scalable photonic circuits $[54,55]$, which are challenging but feasible with integrated quantum photonics in the near future.

The authors thank R. Cao for help during the early stage of this project and X. Jiang for helpful discussions. This research is supported by the National Key Research and Development Program of China (2019YFA0308700, 2017YFA0303704), National Natural Science Foundation of China (Grants No. 11674170, No. 11690032, No. 11321063), NSFC-BRICS (No. 61961146001), Natural Science Foundation of Jiangsu Province (No. BK20170010), the program for Innovative Talents and Entrepreneur in Jiangsu, and the Fundamental Research Funds for the Central Universities.

*Xiaosong.Ma@nju.edu.cn

[1] Y. Aharonov, L. Davidovich, and N. Zagury, Phys. Rev. A 48, 1687 (1993).

[2] J. Kempe, Contemp. Phys. 44, 307 (2003).

[3] A. Ambainis, Int. J. Quantum. Inform. 01, 507 (2003).

[4] N. Shenvi, J. Kempe, and W. K. Birgitta, Phys. Rev. A 67, 052307 (2003).

[5] M. Szegedy, in Proceedings of the Annual IEEE Symposium on Foundations of Computer Science (IEEE, Bellingham, WA, 2004).

[6] S. D. Berry and J. B. Wang, Phys. Rev. A 82, 042333 (2010).
[7] O. Mülken and A. Blumen, Phys. Rep. 502, 37 (2011).

[8] S. D. Berry and J. B. Wang, Phys. Rev. A 83, 042317 (2011).

[9] A. Ahlbrecht, A. Alberti, D. Meschede, V. B. Scholz, A. H. Werner, and R.F. Werner, New J. Phys. 14, 073050 (2012).

[10] J. A. Izaac, X. Zhan, Z. Bian, K. Wang, J. Li, J. B. Wang, and P. Xue, Phys. Rev. A 95, 032318 (2017).

[11] J. A. Izaac, J. B. Wang, P. C. Abbott, and X. S. Ma, Phys. Rev. A 96, 032305 (2017).

[12] A. M. Childs, Phys. Rev. Lett. 102, 180501 (2009).

[13] A. M. Childs, D. Gosset, and Z. Webb, Science 339, 791 (2013).

[14] D. Bouwmeester, I. Marzoli, G. P. Karman, W. Schleich, and J. P. Woerdman, Phys. Rev. A 61, 013410 (1999).

[15] J. Du, H. Li, X. Xu, M. Shi, J. Wu, X. Zhou, and R. Han, Phys. Rev. A 67, 042316 (2003).

[16] A. Schreiber, K. N. Cassemiro, V. Potoček, A. Gábris, P. J. Mosley, E. Andersson, I. Jex, and C. Silberhorn, Phys. Rev. Lett. 104, 050502 (2010).

[17] M. A. Broome, A. Fedrizzi, B. P. Lanyon, I. Kassal, A. Aspuru-Guzik, and A. G. White, Phys. Rev. Lett. 104, 153602 (2010).

[18] A. Peruzzo, M. Lobino, J. C. F. Matthews, N. Matsuda, A. Politi, K. Poulios, X. Q. Zhou, Y. Lahini, N. Ismail, K. Wörhoff, Y. Bromberg, Y. Silberberg, M. G. Thompson, and J. L. OBrien, Science 329, 1500 (2010).

[19] A. Schreiber, A. Gábris, P. P. Rohde, K. Laiho, M. Štefaňák, V. Potoček, C. Hamilton, I. Jex, and C. Silberhorn, Science 336, 55 (2012).

[20] T. Kitagawa, M. A. Broome, A. Fedrizzi, M. S. Rudner, E. Berg, I. Kassal, A. Aspuru-Guzik, E. Demler, and A. G. White, Nat. Commun. 3, 882 (2012).

[21] Y. C. Jeong, C. Di Franco, H. T. Lim, M. S. Kim, and Y. H. Kim, Nat. Commun. 4, 2471 (2013).

[22] X. Qiang, T. Loke, A. Montanaro, K. Aungskunsiri, X. Zhou, J.L. O'Brien, J. B. Wang, and J.C.F. Matthews, Nat. Commun. 7, 11511 (2016).

[23] L. Xiao, X. Zhan, Z. H. Bian, K. K. Wang, X. Zhang, X. P. Wang, J. Li, K. Mochizuki, D. Kim, N. Kawakami, W. Yi, H. Obuse, B. C. Sanders, and P. Xue, Nat. Phys. 13, 1117 (2017).

[24] H. Tang, X. F. Lin, Z. Feng, J. Y. Chen, J. Gao, K. Sun, C. Y. Wang, P. C. Lai, X. Y. Xu, Y. Wang, L. F. Qiao, A. L. Yang, and X. M. Jin, Sci. Adv. 4, eaat3174 (2018).

[25] X. Qiang, X. Zhou, J. Wang, C. M. Wilkes, T. Loke, S. O’Gara, L. Kling, G. D. Marshall, R. Santagati, T. C. Ralph, J. B. Wang, J. L. O'Brien, M. G. Thompson, and J. C. F. Matthews, Nat. Photonics 12, 534 (2018).

[26] G. Zhu, L. Xiao, B. Huo, and P. Xue, Chin. Optic. Lett. 18, 052701 (2020).

[27] H. Schmitz, R. Matjeschk, C. Schneider, J. Glueckert, M. Enderlein, T. Huber, and T. Schaetz, Phys. Rev. Lett. 103, 090504 (2009).

[28] M. Karski, L. Förster, J. M. Choi, A. Steffen, W. Alt, D. Meschede, and A. Widera, Science 325, 174 (2009).

[29] F. Zähringer, G. Kirchmair, R. Gerritsma, E. Solano, R. Blatt, and C. F. Roos, Phys. Rev. Lett. 104, 100503 (2010).

[30] P. M. Preiss, R. Ma, M. E. Tai, A. Lukin, M. Rispoli, P. Zupancic, Y. Lahini, R. Islam, and M. Greiner, Science 347, 1229 (2015). 
[31] Z. Yan, Y. R. Zhang, M. Gong, Y. Wu, Y. Zheng, S. Li, C. Wang, F. Liang, J. Lin, Y. Xu, C. Guo, L. Sun, C. Z. Peng, K. Xia, H. Deng, H. Rong, J. Q. You, F. Nori, H. Fan, X. Zhu, and J. W. Pan, Science 364, 753 (2019).

[32] K. Manouchehri and J. B. Wang, Physical Implementation of Quantum Walks (Springer, Berlin, 2014).

[33] G. D. Paparo and M. A. Martin-Delgado, Sci. Rep. 2, 444 (2012).

[34] G. D. Paparo, M. Müller, F. Comellas, and M. A. MartinDelgado, Sci. Rep. 3, 2773 (2013).

[35] I. Sinayskiy and F. Petruccione, J. Phys. Conf. Ser. 442, 012003 (2013).

[36] T. Loke, J. W. Tang, J. Rodriguez, M. Small, and J. B. Wang, Quantum Inf. Process. 16, 25 (2016).

[37] P. E. Falloon, J. Rodriguez, and J. B. Wang, Comput. Phys. Commun. 217, 162 (2017).

[38] C. M. Bender and S. Boettcher, Phys. Rev. Lett. 80, 5243 (1998).

[39] S. Salimi and A. Sorouri, J. Phys. A 43, 275304 (2010).

[40] T. Loke and J. B. Wang, Ann. Phys. (Amsterdam) 382, 64 (2017).

[41] C. M. Bender and P. D. Mannheim, Phys. Lett. A 374, 1616 (2010).

[42] See Supplemental Material at http://link.aps.org/supplemental/ 10.1103/PhysRevLett.125.240501 for the matrix decomposition, the experimental details, and the quantum-walk advantageous graphs, which includes Refs. [43-48].
[43] M. Reck, A. Zeilinger, H. J. Bernstein, and P. Bertani, Phys. Rev. Lett. 73, 58 (1994).

[44] G. Stewart, SIAM Rev. 19, 634 (1977).

[45] G. W. Stewart, Numer. Math. 40, 297 (1982).

[46] B. D. Sutton, Numerical Algorithms 50, 33 (2009).

[47] I. Dhand and S. K. Goyal, Phys. Rev. A 92, 043813 (2015).

[48] G. R. Fowles, Introduction to Modern Optics (Dover Publications, Inc., New York, 1975).

[49] T. Kim, M. Fiorentino, and F. N. C. Wong, Phys. Rev. A 73, 012316 (2006)

[50] A. Fedrizzi, T. Herbst, A. Poppe, T. Jennewein, and A. Zeilinger, Opt. Express 15, 15377 (2007).

[51] C. K. Hong, Z. Y. Ou, and L. Mandel, Phys. Rev. Lett. 59, 2044 (1987).

[52] A. Peruzzo, M. Lobino, J. C. F. Matthews, N. Matsuda, A. Politi, K. Poulios, X. Q. Zhou, Y. Lahini, N. Ismail, K. Worhoff, Y. Bromberg, Y. Silberberg, M. G. Thompson, and J. L. Obrien, Science 329, 1500 (2010).

[53] H. Wang, J. Qin, X. Ding, M.-C. Chen, S. Chen, X. You, Y.-M. He, X. Jiang, L. You, Z. Wang, C. Schneider, J. J. Renema, S. Höfling, C.-Y. Lu, and J.-W. Pan, Phys. Rev. Lett. 123, 250503 (2019).

[54] J. Wang, F. Sciarrino, A. Laing, and M. G. Thompson, Nat. Photonics 14, 273 (2020).

[55] L. Lu, L. Xia, Z. Chen, L. Chen, T. Yu, T. Tao, W. Ma, Y. Pan, X. Cai, Y. Lu, S. Zhu, and X.-S. Ma, npj Quantum Inf. 6, 30 (2020). 